\title{
X-Ray and Optical Studies of the Thickness Dependence of the Phase Diagram of Liquid- Crystal Films
}

\section{Citation}

Sirota, E. B., Peter S. Pershan, L. B. Sorensen, and J. Collett. 1987. X-ray and optical studies of the thickness dependence of the phase diagram of liquid-crystal films. Physical Review A 36(6): 2890-2901.

\section{Published Version}

doi:10.1103/PhysRevA.36.2890

\section{Permanent link}

http://nrs.harvard.edu/urn-3:HUL.InstRepos:10357473

\section{Terms of Use}

This article was downloaded from Harvard University's DASH repository, and is made available under the terms and conditions applicable to Other Posted Material, as set forth at http:// nrs.harvard.edu/urn-3:HUL.InstRepos:dash.current.terms-of-use\#LAA

\section{Share Your Story}

The Harvard community has made this article openly available.

Please share how this access benefits you. Submit a story.

\section{Accessibility}




\title{
X-ray and optical studies of the thickness dependence of the phase diagram of liquid-crystal films
}

\author{
E. B. Sirota* and P. S. Pershan \\ Department of Physics, Harvard University, Cambridge, Massachusetts 02138 \\ L. B. Sorensen \\ Department of Physics, University of Washington, Seattle, Washington 98195 \\ J. Collett ${ }^{\dagger}$ \\ Division of Applied Sciences, Harvard University, Cambridge, Massachusetts 20138 \\ and IBM Thomas J. Watson Research Center, Yorktown Heights, New York 10598
}

(Received 12 January 1987)

\begin{abstract}
A comprehensive study of the thickness dependence of the phase diagram of freely suspended films of the liquid crystal 4- $n$-heptyloxybenzylidene-4- $n$-heptylaniline (70.7) between 50 and $69^{\circ} \mathrm{C}$ is reported. In thick films (thicker than about 300 layers and characteristic of bulk samples) there is a low-temperature crystalline- $G$ phase followed by five crystalline- $B$ phases with different stacking arrangements at higher temperatures. In thinner films there are two additional crystalline- $B$ phases and two tilted hexatic phases, smectic- $F$ and smectic- $I$, which do not appear in bulk samples. The in-plane and interlayer correlations in the tilted hexatic phases are anisotropic with a clear dependence on the molecular tilt direction; the in-plane correlations are more developed (longer range) perpendicular to the molecular tilt direction and the interlayer correlations are more developed parallel to the tilt direction.
\end{abstract}

\section{INTRODUCTION}

The liquid-crystalline phases of matter, which have order intermediate between that of three-dimensional crystals and fluids, have been extremely fertile systems for studies of the influence of symmetry and dimensionality on phase transitions. Because of their relatively weak interlayer coupling and strong in-plane interactions, these materials can be successfully modeled as a collection of weakly coupled two-dimensional systems. The weak interlayer coupling allows these materials to form threedimensional stacked hexatic phases without spontaneously converting to a three-dimensional crystal. ${ }^{1}$ The freely suspended film technique allows the thickness of the unusual materials to be varied from two molecular layers to thousands of molecular layers, allowing direct experimental studies of the two-dimensional to threedimensional crossover. In addition to probing the effects due to the dimensionality, thin films also provide important information about the influence of the surface field caused by the termination of the material at the surface.

This paper reports our comprehensive studies of the thickness dependence of the phase diagram of freely suspended films of 4- $n$-heptyloxybenzylidene-4- $n$ heptylaniline (70.7). The chemical structure of 70.7 is shown in Fig. 1. The phase diagram is extremely rich-there are 11 distinct bulk phases between 30 and $85^{\circ} \mathrm{C}$ and there are four additional phases that only appear in thin films. ${ }^{2-4}$

The phase sequence of bulk 70.7 was first investigated by Smith and co-workers ${ }^{5,6}$ who found the following sequence: crystal $\rightarrow 33^{\circ} \mathrm{C} \rightarrow$ crystalline- $G \quad(\mathrm{Cr} G) \rightarrow 55^{\circ}$ $\rightarrow$ crystalline- $B \quad(\mathrm{Cr} B) \rightarrow 69^{\circ} \rightarrow$ smectic $-C(\mathrm{SmC}) \rightarrow 72^{\circ}$ $\rightarrow$ smectic- $A(\mathrm{Sm} A) \rightarrow 83.7^{\circ} \rightarrow$ nematic $\rightarrow 84^{\circ} \rightarrow$ isotropic. Subsequent $x$-ray studies of 70.7 and of homologous ${ }^{7-13}$ compounds with similar phase sequences showed that the $\mathrm{Cr} B$ phase in these materials consisted of a series of crystalline phases with different stacking sequences. Motivated by these studies we performed high-resolution $x$-ray scattering measurements on freely suspended films of 70.7 and found that the crystalline- $B$ phase actually consisted of five distinct phases with different stacking sequences: ${ }^{14,15} \quad 55^{\circ} \mathrm{C} \rightarrow$ hexagonal- $A A A \rightarrow 61^{\circ} \rightarrow$ monoclinic - $C \rightarrow 61.5^{\circ} \rightarrow$ orthorhombic $-F \rightarrow 64^{\circ} \rightarrow$ hexagonalclose-packed- $A B A B \rightarrow 69^{\circ}$. We also found that all of these crystalline- $B$ phases, except the hexagonal-closepacked phase, also have a one-dimensional modulation of the smectic layers with the modulation wave vector in the plane of the layers and the polarization normal to the layers. ${ }^{14,15}$ Recently, two of us found that there are also two-dimensional modulations of the smectic layers; these results are reported in the companion paper to this paper. $^{16}$

This paper describes the first comprehensive study of the thickness dependence of phase sequence in freely suspended liquid-crystal films. Our initial expectation that the system would simply cross over from threedimensional to two-dimensional behavior at some small thickness (we guessed two or three layers) greatly underestimated the richness of the behavior we subsequently found-for example, the phase diagram starts to differ from the bulk diagram for films thinner than about 


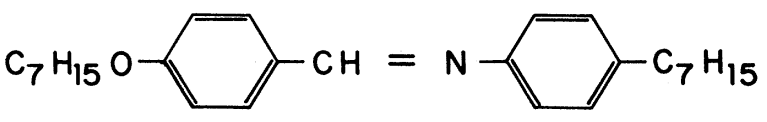

FIG. 1. Chemical structure of 70.7 .

300 layers. We now believe that there is a competition between the effects of the surface field (generated at the film-vacuum interface) and the effects of reduced dimensionality. Some of the observed phase boundaries move to lower temperature as the film thickness is decreased (as expected for reduced dimensionality) and some of the transitions move to higher temperature (as expected for a surface field which favors the ordered low-temperature phase).

\section{EXPERIMENTAL TECHNIQUES}

The thickness dependence of the phase diagram for freely suspended 70.7 films was determined by combining optical microscopy observations with laboratorybased low-resolution x-ray diffraction measurements and with synchrotron-based high-resolution measurements. By combining these techniques, we have been able to study the unusual evolution of the phase diagram for film thicknesses between two molecular layers and about 300 layers where the behavior approaches that of bulk samples.

The films were drawn across a 7-mm-diam. hole in a $0.2-\mathrm{mm}$-thick glass cover slip. The oven has been described in detail previously. ${ }^{15}$ Two modifications were made to improve the performance. (1) The original kapton-X-ray windows were replaced by $0.25-\mathrm{mm}$ bent beryllium windows. This modification produced a substantial reduction in the background scattering from the windows and greatly facilitated the high-resolution x-ray studies of very thin films. (2) The original optical windows were mounted using epoxy. To reduce the straininduced birefringence in the windows, the epoxy seals were replaced by O-ring seals.

Temperature control was provided by a computerinterfaced controller. ${ }^{17}$ Under typical operating conditions ( $\pm 3 \mathrm{~K}$ room-temperature variations) the present single-stage oven provided a temperature stability better than $\pm 5 \mathrm{mK}$ over many hours.

The oven was evacuated and then backfilled with approximately 1 Torr of helium gas. This greatly improved the temperature equilibrium times of the films and produced only a small increase in the $x$-ray scattering background. Permanent magnets did not align the tilt direction of thin hexatic or crystalline phases of 70.7 and therefore were not used.

\section{A. Film-thickness determination}

For very thin films the thickness can be determined very easily by measuring the reflected intensity of a lowpower laser. ${ }^{18}$ For films thinner than about 12 layers, the reflected intensity scales as $N^{2}$, where $N$ is the number of layers. Since $N$ is quantized, a reflected-intensity- versus-film-thickness calibration can be easily obtained by measuring many films. This calibration can be extended up to about 20 layers. For thicker films, the quantized intensity variation gets quite small and it becomes difficult to determine the exact thickness. For thicker films, the thickness can be determined by measuring the optical phase retardation of a transmitted laser beam ${ }^{15}$ or by measuring the apparent reflected color produced for incident white light. The reflected intensity for light of wavelength $\lambda$ and incidence angle $\theta$ from an optically isotropic film with index of refraction $n$ consisting of $N$ layers each of thickness $d$ is given by ${ }^{19}$

$$
I(\lambda, N)=I_{0}(\lambda) \frac{F \sin ^{2} \phi}{1+F \sin ^{2} \phi},
$$

where $I_{0}(\lambda)$ is the incident intensity, $F=4 R /(1-R)^{2}$, and $\phi=(2 \pi / \lambda) N d\left(n^{2}-\sin ^{2} \theta\right)^{1 / 2}$. Although the general expression for the reflectivity from uniaxially anisotropic liquid-crystal films in an arbitrary direction is quite complicated, the special case of $s$-polarized light reflected from a smectic- $A$ film (where the optic axis is perpendicular to the surface of the film) is given by Eq. (1) with $n$ replaced by the ordinary index $n_{0}$ and

$$
R=\left|\frac{\cos \theta-\left(n_{0}^{2}-\sin ^{2} \theta\right)^{1 / 2}}{\cos \theta+\left(n_{0}^{2}-\sin ^{2} \theta\right)^{1 / 2}}\right|^{2} .
$$

For 70.7 films in the smectic- $A$ phase, $n_{0}=1.60,{ }^{20}$ and $d=29.8 \AA ;^{12}$ the angle of incidence for our x-ray film oven is $\theta=25^{\circ}$. To compute the expected colors versus thickness, the intensity distribution $I(\lambda, N)$ was calculated by assuming that the Bausch and Lomb 31-33-05 illuminator produced an incident blackbody spectrum $I_{0}(\lambda)$ with a color temperature of $T=2655 \mathrm{~K}$. The corresponding tristimulus values were computed using the 1931 Commission Internationale de l'Eclairage (CIE) tables $^{21}$ for the tristimulus responses $\bar{x}_{\lambda}, \bar{y}_{\lambda}$, and $\bar{z}_{\lambda}$

$$
\begin{aligned}
X & =\int I(\lambda, N) \bar{x}_{\lambda} d \lambda, \\
Y & =\int I(\lambda, N) \bar{y}_{\lambda} d \lambda, \\
Z & =\int I(\lambda, N) \bar{z}_{\lambda} d \lambda .
\end{aligned}
$$

The resulting tristimulus values were then transformed to chromaticity coordinates $(x$ and $y$ ) and a relative brightness value $(b)$

$$
\begin{aligned}
& x=\frac{X}{X+Y+Z}, \\
& y=\frac{Y}{X+Y+Z}, \\
& b=X+Y+Z .
\end{aligned}
$$

The loci of the calculated chromaticity coordinates versus film thickness are shown on the standard CIE chromaticity diagram in Fig. 2. For films thinner than about 20 layers, this calculation shows that the films will appear gray, becoming whiter (brighter) as the thickness is increased. The color sequence then is orange $(N \simeq 30)$, red $(N \simeq 53)$, blue $(N \simeq 62)$, green $(N \simeq 71)$, yellow $(N \simeq 85)$, red $(N \simeq 113)$, blue $(N \simeq 127)$, green 


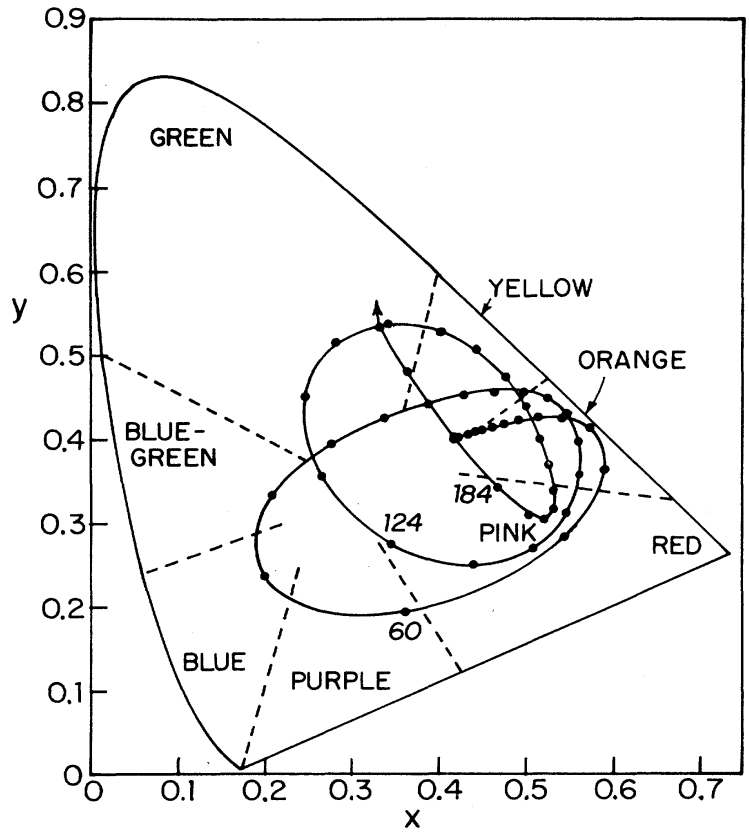

FIG. 2. Calculated chromaticity coordinate $(x, y)$ vs film thickness for 70.7 films with $n_{0}=1.60$ and $d=29.8 \AA$ assuming a $2655-\mathrm{K}$ light source incident at $\theta=25^{\circ}$. The solid line indicates the locus of the chromaticity changes, the dots on the line indicate every fourth layer starting with $N=4$.

( $N \simeq 136)$, yellow ( $N \simeq 153)$, pink $(N \simeq 178)$, pale green, etc. Although the observed colors repeat, the first two loops are sufficiently different that with experience, the orders (for example, the $N=62$ blue and the $N=127$ blue) can be distinguished. Between $N=200$ and 500 layers the color cycles between pale pink, green, and white, becoming paler until the thickest films appear a milky white. This method allowed the thickness to be determined to within $\sim 2$ layers for blue films and to within $\sim 5$ layers for yellow films. This technique is very sensitive to the uniformity of the film; in favorable cases, such as with blue or gray films, film thickness nonuniformities of a single layer can be easily detected. We found it important to monitor the film uniformity since the film occasionally thinned or became nonuniform at several of the phase transitions we studied.

In this study, the reported film thicknesses for films thinner than 15 layers are exact. For films between 16 and 25 layers the uncertainty is \pm 1 layer and for films between 25 and 200 layers the uncertainty is $\pm 5 \%$.

\section{B. Optical observations}

Many of the phase transitions and the macroscopic symmetry of the different phases could be observed qualitatively using polarized optical microscopy. ${ }^{22}$ We used a simple $25 \times$ microscope and crossed polarizers in the specular direction for films illuminated with white light. Since the molecules are very anisotropic, all of the tilted phases (smectic-C, $-F,-I$ and $\mathrm{Cr} G$ ) are strongly birefringent. The phase transitions between nontilted and tilted phases or between the different tilted phases are very easy to observe even in very thin films. By correlating the observed textures with the $x$-ray observations for a few thicknesses, the textures can be identified with specific phases and the thickness-dependent phase diagram can be completed optically. Because of the extreme sensitivity of the optical observations, and because of the marked difference in the textures of the two phases, it is easy to observe the difference between a homogeneous fluid (smectic-C) film and a film with a single hexatic (smectic- $I$ ) overlayer on the two exterior surfaces. Of course $x$-ray measurements are necessary to firmly identify the surface layers as smectic- $I .^{4}$

\section{X-ray measurements}

The low-resolution measurements were performed using the Harvard Materials Research Laboratory $12 \mathrm{~kW}$ Rigaku RU-200 rotating-anode $x$-ray generator operating with an effective source size of $0.3 \times 0.3 \mathrm{~mm}^{2}$ and $\mathrm{Cu}$ $K_{\alpha}$ radiation $(\lambda=1.542 \AA)$. The generator was operated at $4.5 \mathrm{~kW}(50 \mathrm{kV}, 90 \mathrm{~mA})$. The scattering angle $(2 \theta)$

(a)
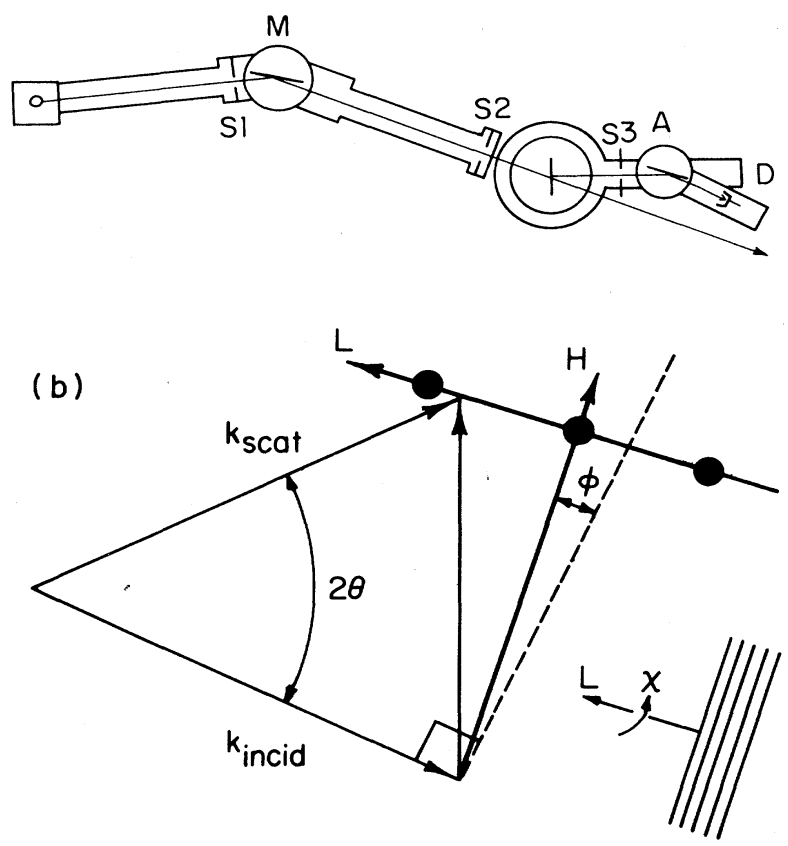

FIG. 3. (a) Low-resolution spectrometer configuration consisting of a bent graphite monochromator (M), a flat graphite analyzer (A), three antiscatter slits $(\mathbf{S} 1, \mathbf{S} 2, \mathrm{~S} 3)$, and a NaI scintillation detector (D). (b) Schematic illustration of the experimental kinematics: $\mathbf{k}_{\text {incid }}$ and $\mathbf{k}_{\text {scat }}$ are the incident and scattered wave vectors, respectively. The reciprocal-lattice vectors $H$ and $L$ are parallel and normal to the lattice planes, respectively. When the angle $\phi=0$, the incident wave vector is normal to the smectic layers. In order to observe scattering from a particular point in the $H-L$ plane, the film must be rotated by an angle $\phi$. The angle $\chi$ defines rotations around the layer normal, $L$. 
and the oven orientation $(\theta)$ was controlled with a Huber 420 two-circle goniometer. The film orientation $(\chi)$ with respect to the scattering plane was controlled with a rotation stage inside the oven. The measurements were made using the geometry shown in Fig. 3. The monochromator was a vertically focusing pyrolytic graphite $(002)$ crystal, 2 in. high with a $115-\mathrm{mm}$ radius of curvature. The source-to-monochromator and monochromator-to-sample distances were equal, producing horizontal Bragg-Brentano parafocusing. The hot spot of the beam at the sample position was $1 \mathrm{~mm}$ wide and $3 \mathrm{~mm}$ high. Because the focusing was imperfect, there were many stray photons which could not be eliminated by.slits $\mathrm{S} 1$ and S2. A brass aperture inside the oven located $1 \mathrm{~mm}$ from the sample on the source side with a diameter $1 \mathrm{~mm}$ less than the $7 \mathrm{~mm}$ diameter of the film prevented the incident photons from hitting the bulk material at the edge of the sample aperture. This was very important for the thin-film studies where the bulk edge scattering would be a serious problem.

The analyzer was a flat $(1 \times 1)$-in. ${ }^{2}$ pyrolytic graphite (002) crystal. To maximize the signal intensity, the detector was placed as close as possible to the sample, $\sim 350 \mathrm{~mm}$. This produced a measured $\chi$ full width at half maximum (FWHM) of $12^{\circ}$ for $2 \theta=20^{\circ}$. The measured zero-arm $2 \theta$ FWHM of the spectrometer was $0.65^{\circ}$ and the measured resolutions for $2 \theta=20^{\circ}$ were $\Delta L=9.2 \times 10^{-3} \AA^{-1}, \Delta H=4.7 \times 10^{-2} \AA^{-1}$. The intensity of the direct beam was $\sim 2 \times 10^{8}$ photons $/$ sec.

The high-resolution studies were done using beam line VII-2 at the Stanford Synchrotron Radiation Laboratory (SSRL). 2,3,23 The oven was oriented with the four-circle Huber 5020 goniometer. The monochromator contained a pair of asymmetrically cut $\mathrm{Ge}(111)$ crystals and the analyzer was a $\operatorname{LiF}(200)$ crystal. The longitudinal resolution was $\Delta H=3.8 \times 10^{-3} \AA^{-1}$ and the transverse resolution was limited by the sample mosaic. The FWHM in $\chi$ was about $2^{\circ}$. For the synchroton studies, the brass aperture inside the oven was unnecessary since the beam was well collimated.

\section{Samples}

Two different commercial sources of 70.7 were used for these studies. ${ }^{24}$ Although the transition temperatures drifted down by as much as $1 \mathrm{~K}$ for some of the material, neither the phase sequence nor any of the observed phase transitions were noticibly affected by the decreased transition temperatures. Recrystallization of the material in petroleum ether restored the original transition temperature. When the sample was maintained in the oven for long periods, the transition temperatures also drifted down. To ensure that the observed thickness dependence of the smectic$(\mathrm{Sm} F)$-to- $\mathrm{Cr} G$ transition was not due to transition temperature drifts, we alternated measurements of thick, thin, and then thick films without reloading the sample; films of the same thickness were measured many times and the measured correlation lengths versus temperature matched when the transition temperatures were shifted to agree. All of the data presented in this study have been corrected (when necessary) to correspond to the unshifted nondegraded transition temperatures.

\section{EXPERIMENTAL RESULTS}

\section{A. Observed phases}

The thickness-dependent phase diagram for 70.7 is surprisingly rich. Nine of the phases in this system are shown in the phase diagram in Fig. 4. Above $69^{\circ} \mathrm{C}$ bulk 70.7 has smectic- $C$, smectic- $A$, nematic, and isotropic phases. The crystalline- $G$ phase persists down to about $33^{\circ} \mathrm{C}$.

The crystalline- $B(\mathrm{Cr} B)$ phases are three-dimensional (3D) crystals ${ }^{7,11,25}$ with the director oriented normal to the layers. The layer spacing $c$ is about $30.6 \AA$ with a small temperature dependence $\left(\sim 2 \times 10^{-4} \AA /{ }^{\circ} \mathrm{C}\right)$. No jumps in the layer spacing were observed at the restacking transitions. ${ }^{16}$ The in-plane nearest-neighbor distance of the hexagonal lattice $a$ is about $5.053 \AA$. In the remainder of this paper we will express the components of the in-plane reciprocal lattice $H$ in units of $4 \pi / \sqrt{3} a=1.4357 \AA^{-1}$ and the normal component $L$ in units of $2 \pi / c=0.2053 \AA^{-1}$. These structures all have very similar in-plane lattice-producing sharp crystalline peaks at $H=1.0$ in these units.

The small temperature- and phase-dependent distortions have been described in detail previously. ${ }^{14-16}$ There are six different $\operatorname{Cr} B$ structures observed in this

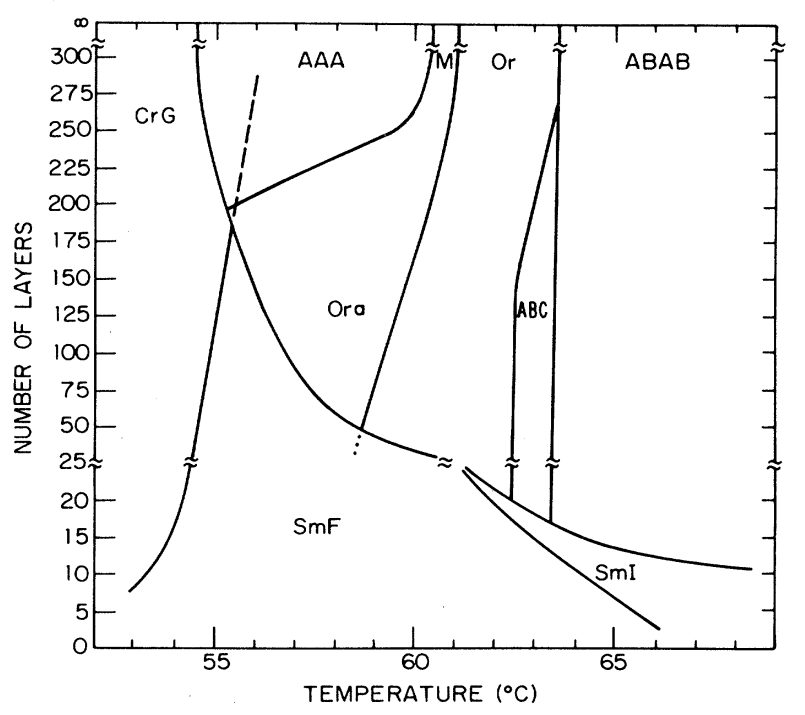

FIG. 4. Thickness vs temperature phase diagram for 70.7 . The solid lines indicate the reversible phase boundaries. The dashed (dotted) lines indicate the phase boundaries of the metastable phases observed kinetically on heating (cooling). The phases are crystalline- $G(\mathrm{Cr} G)$, smectic- $F(\mathrm{Sm} F)$, smectic- $I$ $(\mathrm{Sm} I)$, and the crystalline- $B(\mathrm{Cr} B)$ modifications: hexagonalclose-packed- $A B A B$ ( $A B A B$ ), hexagonal $A B C$ ( $A B C$ ), orthorhombic (Or), orthorhombic-a (Ora), monoclinic (M), and Hexagonal- $A A A(A A A)$. The smectic- $C$ phase $(\mathrm{Sm} C)$ occurs above about $69^{\circ} \mathrm{C}$. 
system. These structures all have very similar in-plane lattices producing sharp crystalline peaks at $H=1.0$. The different interlayer stackings produce peaks at different characteristic $L$ values. The stacking of the thick-film phases has been discussed in detail previously. ${ }^{14,15}$ We report here a new hexagonal- $A B C(A B C)$ and a new orthorhombic (Ora), which only appear in thinner films, and which have not been reported previously. The modulations of the thick-film orthorhombic, monoclinic, and hexagonal- $A A A$ phases are discussed in detail in a companion paper. ${ }^{16}$ Figure 5 shows the locations of the molecules for the different stacking structures, and Fig. 6 shows representative $L$ scans for these phases.

A single-domain $A B A B$ sample has peaks at $L=0$ and $\frac{1}{2}$ [Fig. 6(a)] for all six values of $\chi$ $\left(0^{\circ}, \pm 60^{\circ}, \pm 120^{\circ}, 180^{\circ}\right)$. The observed peaks at $L=\frac{1}{2}$ are approximately three times the intensity of those at $L=0$ as predicted from the structure factor for this stacking. The signature for monodomain $A B C$ samples is $L=\frac{1}{3}$ peaks for $\chi=0^{\circ}, \pm 120^{\circ}$ and $L=\frac{2}{3}$ peaks for $\chi= \pm 60^{\circ}, 180^{\circ}$ [Fig. 6(b)]. Samples with an equal mixture of $A B C$ and $A C B$ stacking will have peaks at $L=\frac{1}{3}$ and $\frac{2}{3}$ for all six $\chi$ positions. $^{20}$ The orthorhombic phase has peaks at $L=0$ for $\chi=0^{\circ}, 180^{\circ}$ and peaks at $L=\frac{1}{2}$ for the other four values of $\chi$ [Fig. 6(c)]. The monoclinic phase has peaks at $L=0$ for $\chi=0^{\circ}, 180^{\circ}$ and at $L= \pm s$ for $\chi= \pm 60^{\circ}, \pm 120^{\circ}$, where $s$ is a temperature-dependent shear parameter with $0<s<0.5$ [Fig. 6(e)]. ${ }^{14}$ The structure and signature of the new Or $a$ phase is discussed below. The $A A A$ phase has peaks at $L=0$ for all six $\chi$

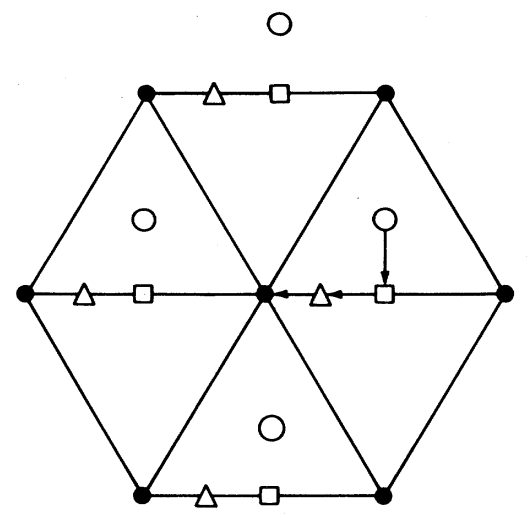

FIG. 5. Relative positions of the molecules in adjacent layers in the different $\operatorname{Cr} B$ phases. The solid dots represent the reference layer positions. The open symbols represent the layer positions in the next layer above for lattices with a singlelayer unit cell. For lattice with a two-layer unit cell, the position of the layer below is the same as that of the layer above. The open circles represent both the hexagonal- $A B C$ structure (one-layer unit cell) and the hexagonal- $A B A B$ structure (twolayer unit cell). The squares indicate the orthorhombic structure positions. The triangles indicate both the monoclinic (one-layer unit cell) and the Ora (two layers per unit cell) structure. In the hexagonal- $A A A$ phase the molecular positions in the adjacent layers are the same as those in the reference layer. values [Fig. 6(d)]. In addition to the peaks listed above, all the phases also have peaks at $L \pm 1$ (actually $L \pm m$ with $m$ an integer), generated by the layer periodicity. The molecular form factor and the Debye-Waller factor produce an overall envelope peaked at $L=0$. At each of the six values of $\chi$, there is a ridge of strong diffuse scattering (with $H=1$ ) due to vibrational modes associated with the layers sliding over each other. ${ }^{15,25,26}$

Similar scans for the crystalline- $G$ phase are shown in Fig. 10. 3, 10,12,27 Peaks are observed at $H=0.925$ for $L=2.6, \chi=0$ and $L=-2.6, \chi=180$ and at $H=0.980$ for $L=1.3, \chi= \pm 60$ and $L=-1.3, \chi= \pm 120$. In addition to these peaks there are also peaks at those $L$ values

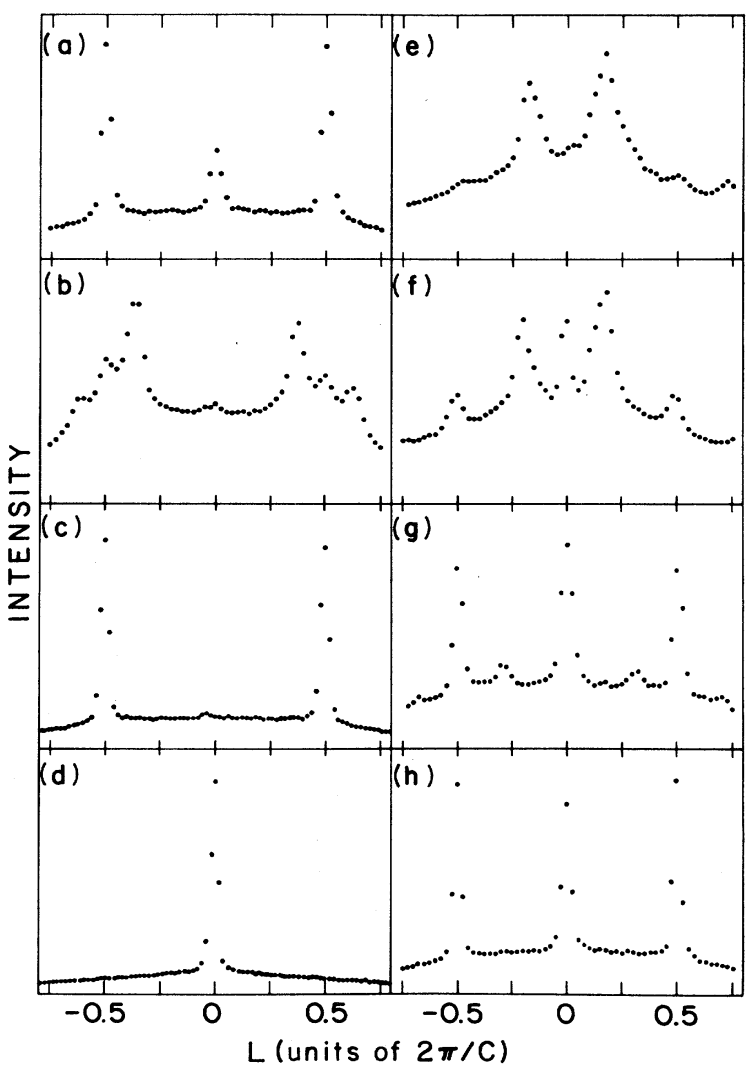

FIG. 6. Typical low-resolution $L$ scans in the different crystalline- $B$ phases. (a) The hexagonal- $A B A B$ phase. The peaks at $L= \pm \frac{1}{2}$ are about three times as strong as the peaks at $L=0$. (b) The hexagonal- $A B C$ phase coexisting with the orthorhombic or the hexagonal- $A B A B$ phase. There are peaks at multiples of $L=\frac{1}{3}$. (c) The orthorhombic phase. There are peaks at $L= \pm \frac{1}{2}$ at four $\chi$ positions $\left(\chi= \pm 60^{\circ}, \pm 120^{\circ}\right)$ as shown. There are also peaks at $L=0$ at the other two $\chi$ positions $\left(\chi=0^{\circ}, 180^{\circ}\right)$, as shown in (d). (d) The hexagonal- $A A A$ phase. There are only peaks at $L=0$. (e) The monoclinic phase in a very thick film. There are peaks at $L=s$ and $L=1-s$. (f) The monoclinic phase coexisting with the Ora phase in a 400-layer film. (g) The orthorhombic phase coexisting with the monoclinic phase in a 200 -layer film. (h) The orthorhombic phase in a 100-layer film. There are peaks at $L= \pm \frac{1}{2}$ and $L=0$. 
plus integral multiples of \pm 1.08 . Like the crystalline- $B$ phases, there are also ridges of strong diffuse scattering. The peaks of the form factor and Debye-Waller factor are at the $H L \chi$ Bragg peak positions, indicating that the molecules are tilted by $\sim 22^{\circ}$. (The tilt varies from $22^{\circ}-24^{\circ}$ with temperature.) The crystalline- $G$ phase is a 3D crystal with the molecules stacked end to end; the tilt of the primitive translation vectors coincides with the molecular tilt. The molecular tilt direction is towards the face of the hexagon formed by its nearest inplane neighbors. The lattice parameters ${ }^{12}$ are $a=9.45$ $\AA, b=5.15 \AA, c=30.6 \AA$, and $\beta=111.9^{\circ}$.

The diffuse peaks in the smectic- $F$ phase peak $^{3,27-31}$ occur at the same positions as the crystalline peaks of the crystalline- $G$ phase. The interlayer correlation lengths determine the width along $L$ and the in-plane correlation lengths determine the width along the $H$ direction. The peaks are located in the same positions in these two phases because they have the same local packing. ${ }^{32}$ The $\mathrm{Sm} F$ phase is a stacked tilted hexatic phase ${ }^{33}$ with short-range in-plane positional order; the in-plane positional correlations decay exponentially with a decay length of about 50 molecular diameters.

The smectic- $I$ phase $\mathrm{e}^{3,30,29,34-36}$ is a stacked tilted hexatic phase, with the molecular tilt direction toward the corner of the hexagon formed by its nearest neighbors. The diffuse peaks appear at $H=0.988, L=0, \chi=0,180$; $H=0.955, L=2, \chi=60,120$; and $H=0.955, L=-2$, $\chi=240,300$. The crystalline analogue of the smectic- $I$ phase is the crystalline- $J$ phase, which does not occur in 70.7. The lattice parameters for the smectic-I structure are $a=5.34 \AA, b=8.86 \AA, c=30.6 \AA$, and $\beta=109^{\circ}$.

\section{B. Restacking transitions}

Below about 200 layers, the hexagonal- $A B C$ phase appeared between the hexagonal- $A B A B$ and the orthorhombic phases. This was a very difficult region in the phase diagram to study because the films tended to rupture in the $A B C$ phase. Films about 60 layers thick ruptured soon after the $A B C$ phase formed (either by cooling or heating). Films over 100 layers thick were stable longer; one 200-layer film was stable for days. However, in the stable films, the $A B C$ structure coexisted with either the orthorhombic or the $A B A B$ structure. The films did not break if they were cooled or heated quickly through the $\mathrm{ABC}$ temperature range. A typical $L$ scan for the coexisting $A B C$ structure is shown in Fig. 6(b). The insertion of the $A B C$ phase between the $A B A B$ and the orthorhombic phase, produces the same phase sequence as that found in bulk $n$-[4-( $n$-pentyl)oxybenzylidene]- $4^{\prime}-\left(n\right.$-hexyl)aniline $(50.6){ }^{37}$

In films thicker than $\sim 300$ layers, the monoclinic phase $^{15}$ was always observed between the orthorhombic and the $A A A$ phases [Fig. 6(e)]. However, weak peaks at $L= \pm \frac{1}{2}$ were always observed in the monoclinic phase. ${ }^{37}$ These peaks became weaker and disappeared as the temperature was lowered towards the $A A A$ phase. Below about 150 layers, the transition between the orthorhombic and the monoclinic phase gradually changed, until it occurred without any evidence for peaks at the $L$ values associated with the monoclinic phase [Fig. 6(h)]. The x-ray signature for this transition was that the orthorhombic domains with peaks at only $L= \pm \frac{1}{2}$ developed peaks at $L=0$. As the temperature was lowered, the $L= \pm \frac{1}{2}$ peaks continuously weakened and the $L=0$ peak continuously strengthened, until only the $L=0$ peak remained. The orthorhombic domains with peaks at $L=0$ remained unchanged. Between about 150 and 300 layers, this continuous orthorhombic-to-hexagonal- $\boldsymbol{A} A \boldsymbol{A}$ transition coexisted with small monoclinic peaks [Fig. $6(\mathrm{~g})$ ].

This transition can be interpreted either as a new intermediate orthorhombic- $a$ phase (Ora) between the orthorhombic and the hexagonal- $A A A$ phases or as a coexistence between the hexagonal- $A A A$ and the orthorhombic phases. In the monoclinic phase the displacement of each layer with respect to the one below it occurs in the same direction. This results in a tilted monoclinic structure with one molecule per unit cell. The observed $x$-ray scattering from the new Or $a$ phase is consistent with a structure produced by displacing the layers in alternating directions so that the macroscopic symmetry is orthorhombic. The resolution-limited peaks at both $L=0$ and $\pm \frac{1}{2}$ for the same $\chi$ position require an Ora unit cell with at least two molecules per unit cell. The real-space structures for the observed crystalline- $B$ phases are shown schematically in Fig. 7; the monoclinic and the Ora structures have the same local packing between adjacent layers, but differ in their second-neighbor packing. The weak $L=\frac{1}{2}$ peaks in the thick films may be due to Ora surface layers on the exterior surfaces of the monoclinic interior. The observed $x$-ray intensities are consistent with about 75 Ora layers on each surface.

The second interpretation of the data is that there is a coexistence of the orthorhombic and the $A A A$ phases. High-resolution x-ray measurements of an 80-layer film showed that the $L$ widths of the Bragg peaks were limited only by the 80-layer finite thickness, and did not broaden as the peak intensities diminished. Thus if there is coexistence, it must occur in the direction parallel to the film surface and the lateral domain size of the coexisting domains must be smaller than the $(1 \times 2)-\mathrm{mm}^{2}$ size of the beam. Since this effect was observed in different batches of 70.7 we tend to rule out impurities as the cause of the coexistence. While we cannot absolutely rule out coexistence, we have no explanation for its appearance.

The optical observations (with crossed polarizers) showed that the crystalline- $B$ phase, produced by cooling from the smectic- $C$ phase, initially formed without any visible observable texture. However, as the temperature was lowered, a weak birefringent texture (much weaker in contrast than the $\mathrm{SmC}$ or the lowertemperature tilted phases) was observed. This texture became progressively stronger as the temperature was lowered. This texture is apparently due to the formation of tilted surface layers on the two exterior surfaces, as originally noted by Farber $^{38}$ - on close inspection the observed tilt domains clearly overlap, indicating two distinct sets. The actual phase of the surface layers has not been determined; the optical texture could be caused by 
(a)

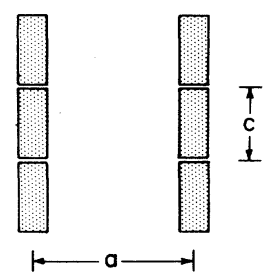

(c)

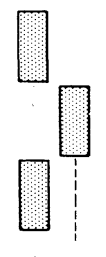

|sa

(e)
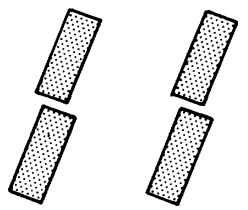

(d)
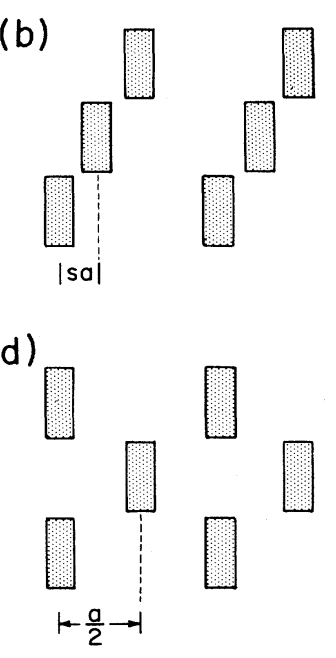

FIG. 7. Schematic view of the projection of the molecular axes onto the (100) plane showing the in-plane projections for the (a) hexagonal- $A A A$ phase, (b) monoclinic phase, (c) orthorhombic phase, (d) Ora phase, and (e) the crystalline- $G$ phase. The angle between the layer normal and the primitive translation vector is $\gamma$ and is determined from the position of the Bragg peak. The angle $\beta$ between the long molecular axis and the layer normal is somewhat model dependent, however, as discussed in the text, measurements indicate that the two are indistinguishable.

smectic- $F$, smectic- $I$, crystalline- $G$, or crystalline- $J$ surface layers. An $x$-ray study to determine the surface phase is planned. We recently used x-ray methods to demonstrate the formation of surface smectic- $I$ layers on the two exterior surfaces of 70.7 smectic-C films. ${ }^{4}$

\section{Tilted phases}

Using the low-resolution spectrometer at Harvard the thickness dependence of the smectic- $F$-to-crystalline- $G$ transition was studied versus film thickness by measuring the x-ray scattering profiles along the $L$ direction. ${ }^{3}$ This spectrometer allowed measurements of the interlayer correlations for films thicker than about eight layers. The longitudinal resolution of this spectrometer was too broad to allow measurements of the in-plane positional evolution. The difference in optical textures between the smectic- $F$ and crystalline- $B$ phases made it quite easy to visually-determine the phase boundary between the two phases for films thicker than about 14 lay-

ers. In thinner films, there was less contrast and the change at the transition was quite subtle, making it difficult to determine the transition optically. Consequently, for thinner films the $\mathrm{x}$-ray signature (the diffuse peaks in the smectic- $F$ phase becoming resolution- and finite-size-limited Bragg peaks in the crystalline- $G$ phase) was used to establish the phase boundary. The composite transition temperature versus film thickness diagram is shown in Fig. 4. At first there is a gradual decrease in the transition temperature as the thickness is reduced, then below about 25 layers there is a substantial decrease.

The interlayer correlations were studied using $L$ scans through the peaks at $L= \pm 2.6$ and $L= \pm 1.3$; the peaks at $L= \pm 1.3$ were more than twice as wide as those at $L= \pm 2.6$, indicating an anisotropy in the interlayer disorder correlated with the molecular tilt direction. Typical $L$ scans at these positions are shown in Fig. 9. The anisotropy is presumably produced by the anisotropic steric interactions produced by the molecular tilt. A remnant of the anisotropy is also visible in the diffuse $\mathrm{Cr} G$ scattering as shown in Fig. 10. The diffuse scattering ridge produced by the soft modes corresponding to the layers sliding over each other is approximately twice as strong (relative to the Bragg peaks) near the $L= \pm 1.3$ peaks as it is near the $L= \pm 2.6$ peaks.

The temperature dependence of the interlayer correlations was also studied. With our low-resolution spectrometer, it was much easier to study the $L= \pm 2.6$ peaks than the $L= \pm 1.3$ peaks. The low-resolution measurements presented here are for the $L= \pm 2.6$ peaks. Because the samples were always unoriented $\chi$ powders, the appropriate line shape is a square-root Lorentzian (SRL) profile. ${ }^{39,40}$ The data were fit to independent SRL's at $L=2.63 .68$, and 1.52 multiplied by the Gaussian envelope produced by the molecular form factor. A background term was also included. The measured smectic- $F$ interlayer correlation length versus temperature and film thickness is shown in Fig. 11. The observed correlation lengths are independent of the film thickness and exhibit identical temperature dependence. However, in thinner films the $\mathrm{Sm} F$-to- $\mathrm{Cr} G$ transition occurs at a lower temperature and the $\operatorname{Sm} F$ correlations develop further before the transition occurs. The ob-

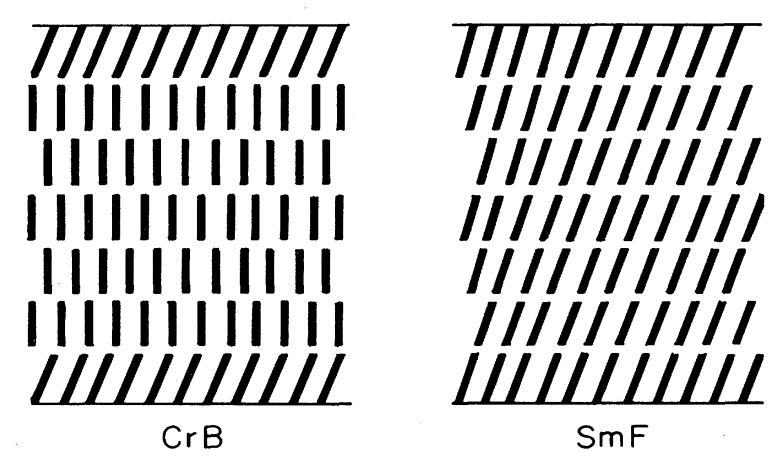

FIG. 8. Proposed structures of the smectic- $F(\mathrm{Sm} F)$ and of the crystalline- $B(\mathrm{Cr} B)$ with tilted surface layers. 


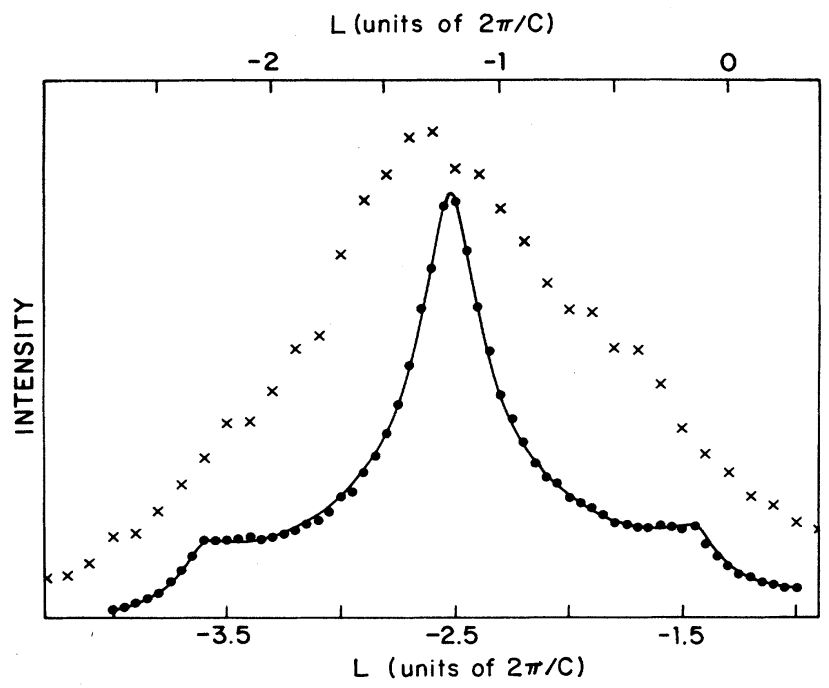

FIG. 9. Typical $L$ scans in the $\operatorname{Sm} F$ phase for a 125-layer film at $T=55^{\circ} \mathrm{C}$. Scans through the $L=-2.6$ peak are shown by dots, scans through the $L=-1.3$ peaks are shown by crosses. The solid line is the fit to square-root Lorentzian peaks centered at $L=-2.6,-3.68$, and -1.52 multiplied by the Gaussian envelope produced by the molecular form factor. These data were collected with the low-resolution spectrometer. The peak counting rates were 300 counts/sec for $L=-2.6$ and 100 counts/sec for $L=-1.3$.

served temperature dependence appears to follow a universal curve; extrapolation of this curve to thinner films than we have measured indicates that the $\mathrm{SmF}$-to$\mathrm{Cr} G$ transition may change from first order to second order or be continuous for films thinner than about four layers. Unfortunately, this could not be studied with the low-resolution spectrometer, since, as shown in Fig. 11, the widths along the $L$ direction eventually became dominated by the finite film thickness in thin films. The inplane correlations can be measured using the highresolution synchrotron based spectrometer, and we plan to study this transition in thin films in the future. (See Fig. 13.)

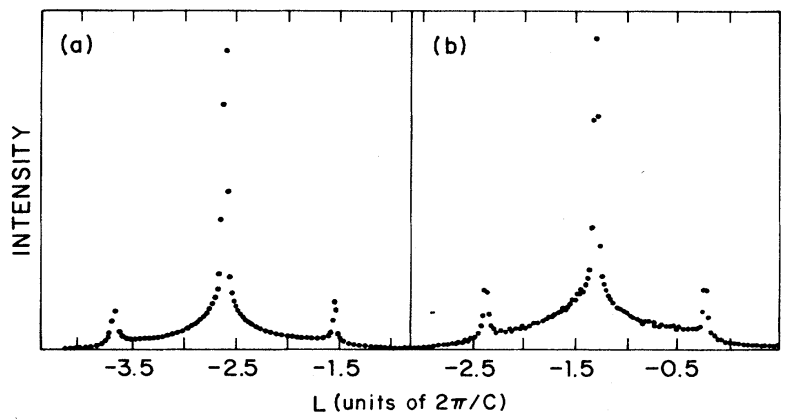

FIG. 10. Low-resolution $L$ scans through the (a) $L=-2.6$ and (b) $L=-1.3$ peaks in the crystalline- $G$ phase. The weaker secondary peaks offset by \pm 1.08 in $L$ are visible. The relative intensity of the diffuse ridge is about twice as strong for the $L=-1.3$ peak as it is for the $L=-2.6$ peak.

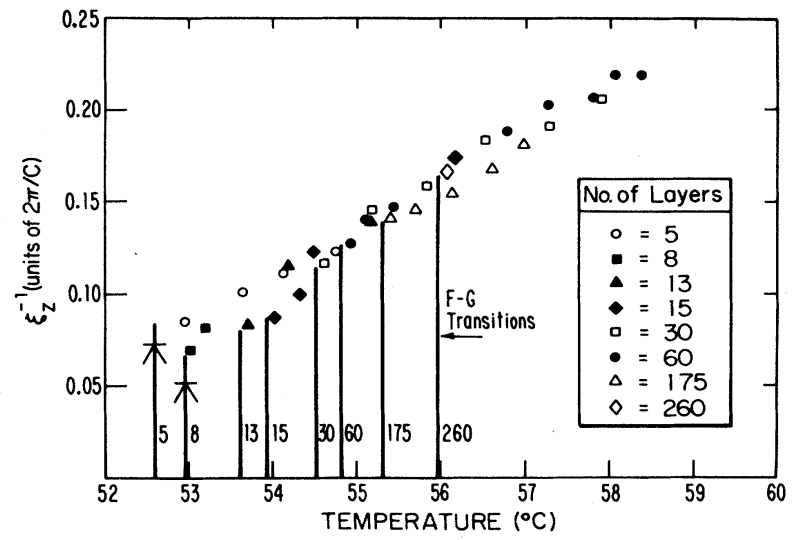

FIG. 11. Observed inverse correlation lengths from the low-resolution measurements of the temperature dependence of the $L$ linewidths for the $L=2.6$ peak of the smectic- $F$ phase of 70.7 vs film thicknesses $N$. For each thickness, the widths follow a universal curve until they undergo the first-order jump (indicated by the solid bar) at their respective smectic$F$-to-crystalline- $G$ transitions. The two arrows indicate the finite-size limits for five- and eight-layer films, respectively.

In thicker films, our previous high-resolution measurements have shown that there is a large pretransitional evolution of the in-plane and the interlayer correlations followed by a small first-order jump at the $\mathrm{Sm} F$-to-Cr $G$ transition. The observed linewidths for 13-layer films are shown in Fig. 12. In addition to the interesting pre-

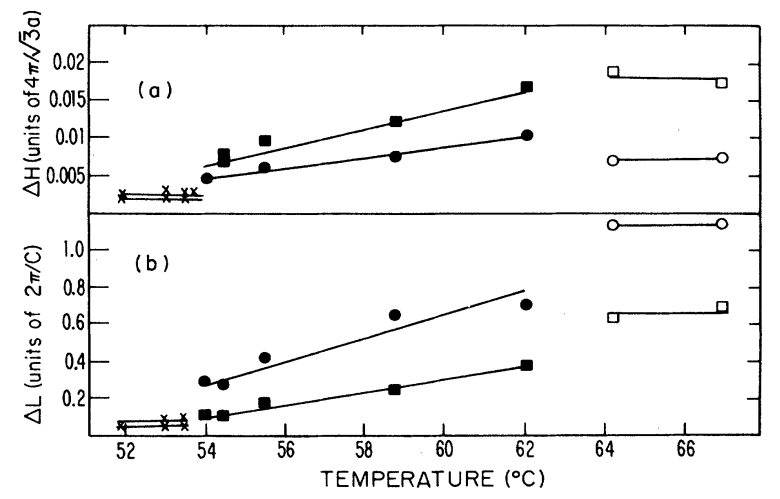

FIG. 12. Temperature dependence of the measured linewidths along the $H$ direction (a) and along the $L$ direction (b) in the crystalline- $G$, smectic- $F$, and smectic- $I$ phases of 70.7, taken with the high-resolution spectrometer at SSRL for a 13-layer film. The solid lines are guides for the eye. SmI phase: Open circles indicate $H=0.988, L=0$; open squares indicate $H=0.955, L=2 . \mathrm{Sm} F$ phase: Closed circles indicate $H=0.975, L=1.3$; closed squares indicate $H=0.925, L=2.6$. (a) Anisotropic in-plane linewidths [half widths at half maximum (HWHM)] from the $H$ scans. The crosses indicate the instrumental resolution measured in the $\mathrm{Cr} G$ phase. The widths are presented in $H$ units. (b) Linewidths (HWHM) illustrating the anisotropic interlayer correlations from the $L$ scans. The crosses indicate the combined mosaic and finite-size limit, measured in the $\mathrm{Cr} G$ phase. The widths are presented in $L$ units. 
transitional correlation length evolution, these data also show that both the in-plane and the interlayer correlations of the smectic- $F$ and the smectic- $I$ phases are anisotropic. In both cases, the peaks closest to the tilt direction $(\mathrm{Sm} F L= \pm 2.6, \operatorname{Sm} I L= \pm 2)$ are broader in the in-plane direction and narrower in the interlayer direction than the peaks farthest from the tilt direction ( $\mathrm{Sm} F L= \pm 1.3, \mathrm{Sm} I L=0)$. This anisotropy is consistent with the steric anisotropy produced by the tilt.

Previous measurements by Doucet and Levelut ${ }^{12}$ of the crystal structure (giving the lattice tilt $\gamma$ ) and the layer spacing (giving a measure of the effective molecular tilt $\beta$ ) showed that the two tilts were the same to about $1^{\circ}$; when $\gamma=\beta$ the molecules are packed end to end. We have investigated the packing in the smectic- $F$ and smectic-I phases of 70.7. The angle between the reciprocal-lattice-vector direction (with orientation $\phi$ and $2 \theta$ ) and the smectic-layer direction is $\phi-\gamma=(2 \theta) / 2$. The molecular tilt direction $\beta$ can be determined quite accurately by measuring the difference in the $L$ values between primary and secondary $L$ peaks since $\beta=\cos ^{-1}(2 / \Delta L)$. Typical data for the $L-2.6$ $\mathrm{Cr} G$ primary-to-secondary-peak separation were $\Delta L=2.146 \pm 0.005$, yielding $\beta=21.3 \pm 0.3$ and a corresponding $\gamma$ value of $\gamma=\phi-(2 \theta) / 2=21.6 \pm 0.1^{\circ}$. The molecular tilt and the layer tilt are coincident to $\pm 0.4^{\circ}$ in the $\mathrm{Cr} G$ phase are presumably in both the smectic $F$ and the smectic- $I$ phases.

We also investigated the molecular packing of the crystalline- $G$ phase. High-resolution measurements of the Bragg peaks showed that magnitude of the reciprocal-lattice vector of the $L=2.6$ peak was greater than that of the $L=1.3$ peak by $0.75 \% \pm 0.02 \%$. Assuming that the molecules are packed end to end (with $\gamma=\beta)$, this requires a closer packing, in the plane normal to the long molecular axes, of the molecules along the tilt direction than in that perpendicular to the tilt direction, by $0.75 \% .^{41}$ Similar results have been reported in other studies. ${ }^{10,12,35,42}$ If this effect were due to the tilt of the molecules $(\beta)$, it would require that $3 / 8\left[\cos ^{2}(\gamma) / \cos ^{2}(\beta)-1\right]=0.0075$ or $\beta-\gamma=1.5^{\circ}$. Our data show that a difference between the two tilt angles cannot be responsible for the measured anisotropy. The observed packing anisotropy is probably caused by the tendency for local herringbone packing produced by the planar geometry of the benzene rings in the molecule. However, there is no long-range herringbone order in the $\mathrm{Cr} G$ phase. When herringbone order is observed, the resulting distortion is very pronounced. ${ }^{42}$

The evolution of the tilt in the $\operatorname{Sm} I, \operatorname{Sm} F$, and $\mathrm{Cr} G$ phases was determined from the inclination of the peaks relative to the plane of the layers. With decreasing temperature the tilt increased continuously from its value of $\sim 19^{\circ}$ in the $\mathrm{SmI}$ phase at a rate of about 0.5 degrees $/ \mathrm{K}$. For films thicker than about 10 layers there was no observed thickness dependence of the tilt. The tilt evolution at the $\mathrm{Sm} F$-to-SmI transition appeared to be continuous, consistent with previous work. ${ }^{34}$

Optically, the $\mathrm{Sm} F$ and $\mathrm{SmI}$ phases have the same texture. The $\mathrm{SmI}$-to-Sm $F$ transition is visible because the domains change their relative brightness, corresponding

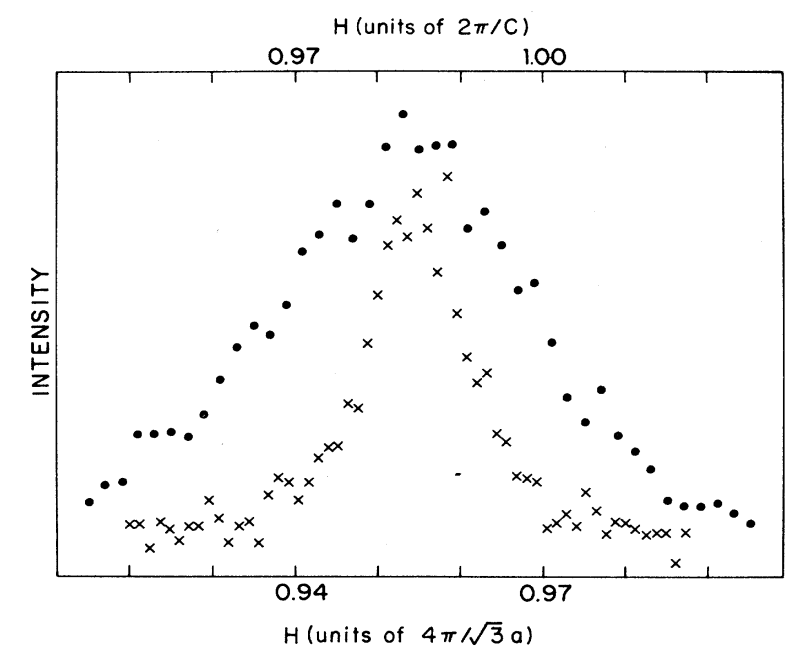

FIG. 13. $H$ scans in the $\operatorname{Sm} I$ phase of a 13-layer film at $T=62.3^{\circ} \mathrm{C}$. The $L=2$ data are shown by dots and the $L=0$ data are indicated by crosses. These data were collected at SSRL. The peak counting rate was about 200 counts per minute.

to the change in the tilt direction at the transition. ${ }^{22} \mathrm{Al}-$ though these two phases cannot be distinguished optical$1 y$, the combination of a few x-ray measurements with an optical determination of the phase boundary provides a complete determination. The $\mathrm{Sm} F$-to-SmI phase boundary shown in Fig. 4 was determined with combined optical and x-ray measurements.

\section{Nontilted-to-tilted phase transitions}

Optical and $\mathrm{x}$-ray observations of the transitions from the nontilted crystalline- $B$ phase to the tilted $\operatorname{SmI}, \operatorname{Sm} F$, and $\mathrm{Cr} G$ phases were made. In bulk 70.7 , there is a direct, $\mathrm{Cr} B$-to- $\mathrm{Cr} G$ transition at about $54.5^{\circ} \mathrm{C}$. In films thinner than about 180 layers, there is a $\operatorname{Sm} F$ phase between the $\operatorname{Cr} B$ and the $\operatorname{Cr} G$ phases. As shown in Fig. 4, the $\mathrm{Sm} F$-to- $\mathrm{Cr} B$ transition temperature increases with decreasing thickness. Below about 20 layers there is also a $\mathrm{SmI}$ phase. The $\mathrm{SmI}$ phase first appears between the $\mathrm{Cr} B$ and the $\mathrm{Sm} F$ phases in films about 20 layers thick. As the thickness is reduced, the $\mathrm{SmI}$ range grows and the $\operatorname{Cr} B$ range shrinks until about 10 layers where the $\mathrm{Cr} B$ phase disappears and there is a direct $\mathrm{SmC}$-to-SmI transition. The transitions between the $\operatorname{Cr} B$ phase and the lower-temperature tilted phases were all found to be first order with significant hysteresis.

In contrast to the $\mathrm{Cr} B$ restacking transitions and the $\mathrm{Sm} F$-to-Cr $G$ transition which were observed to take minutes to occur, the transitions between the $\mathrm{Cr} B$ phases and the tilted phases all occurred very slowly (hours). By performing x-ray scans rapidly, after the oven temperature equilibrated but before the sample reached its equilibrium structure, it was possible to map out the reversible $\mathrm{Cr} B$ restacking transitions at temperatures where the equilibrium structure was $\operatorname{Sm} F$. These transient phase boundaries are indicated on the phase dia- 
gram (Fig. 4) by dotted lines. Similarly, by performing rapid scans while heating out of the $\operatorname{Cr} G$ phase it was possible to observe a reversible $\mathrm{Cr} G$-to-Sm $F$ transition in the temperature range in which the equilibrium structure was $\mathrm{Cr} B$. This is indicated by the dashed line in Fig. 4.

It is possible to construct a simple model which correctly describes the observed thickness dependence of the $\mathrm{Cr} B$-to-Sm $F$ transition temperature. Because the optical observations show that there are tilted molecules on the two free surfaces of the $\operatorname{Cr} B$ films (see Fig. 8), we assume that the surface field produces $\operatorname{Sm} F$ surfaces. Let $N$ denote the total number of layers in the film and let $n$ denote the number of surface layers $(2 \leq n \leq N)$. The excess free energy per surface layer is $F(T)=F_{F}(T)-F_{B}(T)$ where $F_{F}(T)$ and $F_{B}(T)$ are the free energies per layer of the $\operatorname{Sm} F$ and $\operatorname{Cr} B$ phases. We assume the simplest form for the temperature dependence, of the excess free energy, $F(T)=a\left(T-T_{c}\right)=a t$, where $t$ is the reduced temperature and $T_{c}$ is the temperature where a bulk $\mathrm{Cr} B$-to-Sm $F$ transition would occur if the $\mathrm{Cr} G$ phase did not intervene. We also assume that the two surfaces are always forced to be tilted by the surface field (consistent with the optical observations). The total free-energy cost for the tilted surface layers is then $n a t+F_{w}$, where $F_{w}$ is the energy of the wall between the tilted surface phases and the nontilted interior. We assume a constant wall cost of $c / 2$ per wall, so that

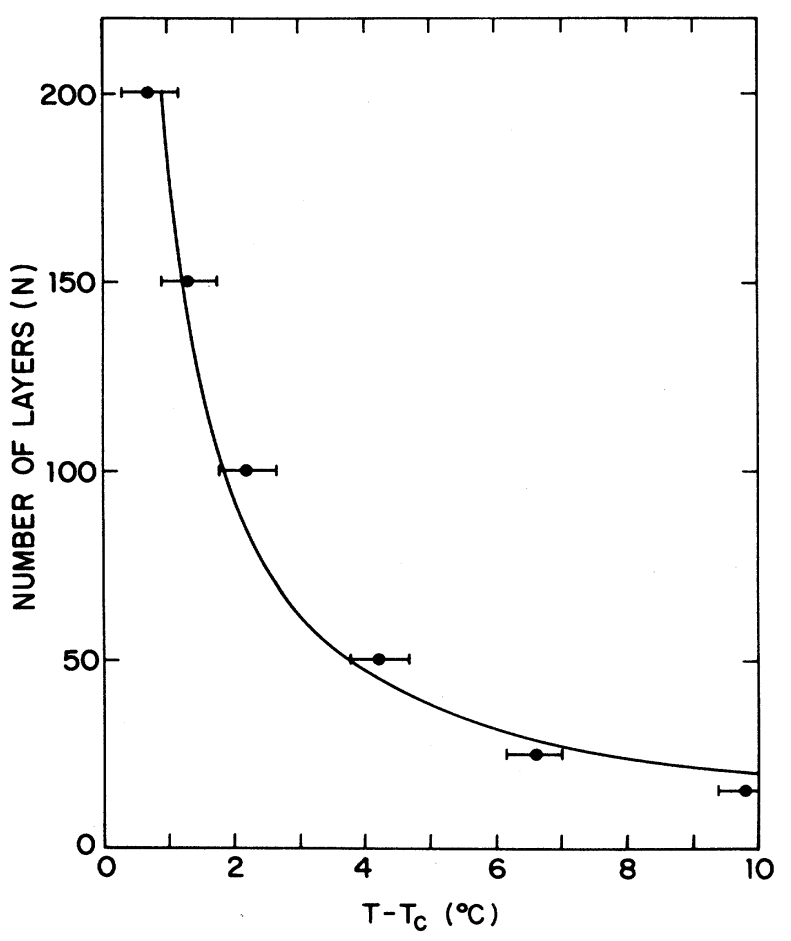

FIG. 14. Predicted thickness dependence (solid line) of the smectic- $F$-to- crystalline- $B$ phase boundary compared to the observed transition temperatures (data with error bars). The model parameters are $c / a=180 \mathrm{~K}$ and $T_{c}=54.5^{\circ} \mathrm{C}$.
$F_{w}=c$, and note that the minimum surface cost occurs for two surface layers and is equal to $2 a t+c$. Comparing this cost to the cost of converting the entire film to the tilted form ( $\mathrm{Nat}$ ) shows that the relative energy cost is $\Delta=-(N-2) a t+c$. This model predicts a transition when the relative cost vanishes, $\Delta=0$, or $t=c / a(N-2)$. The agreement of this prediction with the observed $\operatorname{Cr} B-\operatorname{Sm} F$ phase boundary is shown in Fig. 14 for $c / a=180 \mathrm{~K}$ and $T_{c}=54.5^{\circ} \mathrm{C}$.

More detailed models might explain the observed increasing birefringence in the $\mathrm{Cr} B$ phase as the temperature is lowered. We have not pursued this further since we do not have sufficient data to distinguish between increased tilt of single surface layers and an increased number of tilted surface layers. Further $x$-ray and optical studies are planned.

\section{DISCUSSION}

The observed anisotropy of the in-plane and interlayer correlation lengths in the tilted hexatic phases must be produced by a combination of the intrinsic molecular anisotropy and the tilted hexatic structure. The peaks closest to the tilt direction have smaller in-plane correlation lengths than the peaks that are closer to the perpendicular direction. This is consistent with simple steric considerations which imply that the intralayer lattice spacing in the direction closest to the tilt will be more strongly affected by tilt-orientation fluctuations producing in-plane decorrelation. In addition, the homogeneous relative motions between adjacent layers along the direction of the tilt are suppressed, producing larger relative layer displacements perpendicular to the tilt direction than parallel to it. This produces the decreased $L$ widths for the nearly perpendicular peaks.

As noted in the Introduction, some of the observed phase boundaries move to lower temperatures with decreasing film thickness and some of the phase boundaries move to higher temperature. The crystalline$B$-to-smectic- $F$ transition moves to higher temperature as the film thickness is decreased. This is an unusual transition because the low-temperature phase, smectic- $F$ which is a tilted hexatic, is less positionally ordered than the high-temperature phases, crystalline- $B$ which is a nontilted crystal. As the simple model in Sec. III demonstrates, this is exactly the kind of behavior expected for a surface field which favors tilted molecules at the film-vacuum interface. Very similar behavior has been observed for the smectic- $A$-to-smectic- $C$ transition in thin films ${ }^{43}$ where the surface field is also believed to favor tilt at the surface. There is a number of liquid crystal systems where the surface field produces a more ordered surface phase, including the formation of nontilted crystalline- $B$ surface layers on smectic- $A$ films, ${ }^{39,44,45}$ the formation of surface tilted hexatic smectic- $I$ layers on smectic- $C$ films, ${ }^{4}$ and the production of surface-induced smectic- $A$ order at the surfacevacuum interface of bulk nematic or isotropic samples. ${ }^{46,47}$

The observed decrease in the transition temperature with decreasing film thickness observed for the smectic- 
$F$-to-crystalline- $G$ transition is consistent with the predicted suppression of the ordered phase in two dimensions due to the increased importance of the thermal fluctuations. To our knowledge this is the first observation of a suppressed transition in a liquid-crystal system. Since the local order in the smectic- $F$ and crystalline- $G$ phases are the same, this transition is allowed by symmetry to be second order. Because the films are freely suspended there is no periodic substrate to influence the transition, and this transition should be a superb model for the two-dimensional tilted hexatic to tilted crystal transition. ${ }^{48,49}$ The observed decrease in the size of the first-order jump with film thickness suggests that this transition might become second order for thicknesses below about four layers, with a universal correlation length evolution versus temperature in the hexatic phase.

It seems very likely that there will be similar restacking sequences and thin-film hexatic phases in many liquid-crystal materials. We have made measurements for the two 70.7 homologos, $n$-[4-( $n$-pentyl)oxybenzylidene $]-4^{\prime}-(n$-hexyl)aniline $\quad(50.6)$ and $n$-[4- $(n-$ nonyl)oxybenzylidene]-4'-( $n$-butyl)aniline (90.4), which exhibit bulk hexatic (smectic-F) phases. Both of these materials have a correlation-length anisotropy similar to that discussed above for 70.7. In addition, the smectic$F$-to-crystalline- $G$ phase boundary of 90.4 shows qualitatively the same suppression with decreasing thickness as 70.7. The crystalline- $B-$ to-smectic- $F$ phase boundary of 50.6 also has qualitatively the same thickness dependence as 70.7. The observed thin-film restacking sequence in $70.7 \quad(A B A C \rightarrow A B C \rightarrow$ orthorhombic $\rightarrow$ monoclinic $\rightarrow A A A$ ) is also observed in bulk 50.6 films, ${ }^{20}$ and except for the final $A A A$ phase, in another homolog (4O.8) which has crystalline- $B$ phases (90.4 does not).

In conclusion, the striking changes in the phase sequence and phase boundaries of 70.7 (and similar) films versus film thickness provide a wealth of information about the influence of reduced dimensionality and the strong surface fields on these systems. The smectic$F$-to-crystalline- $G$ transition, which exhibits a suppressed transition temperature with decreasing thickness, appears very promising for future two-dimensional melting studies in a substrate free system. The crystalline- $B$-to-smectic- $F$ transition is well described by a simple surface field model.

\section{ACKNOWLEDGMENTS}

We wish to acknowledge helpful conversations with Allan Farber, B. I. Halperin, D. R. Nelson, and D. E. Moncton. We also wish to acknowledge the help of Kelby Chan, Frank Molea, Alan Braslau, George Pisiello, and the staff at SSRL. This work was supported by the National Science Foundation under Grant No. DMR85-13523 and DMR-80-20247. Part of this work was done at the Stanford Synchroton Radiation Laboratory, which is supported by the U.S. Department of Energy, Office of Basic Sciences.
*Present address: Corporate Research Science Laboratories, Exxon Research and Engineering Company, Route 22 East, Annandale, NJ 08801.

${ }^{\dagger}$ Present address: IBM, Department 242/040-2, Rochester, MN 55901.

${ }^{1}$ R. J. Birgeneau and J. D. Litster, J. Phys. (Paris) Lett. 39, L399 (1978).

${ }^{2}$ J. Collett, P. S. Pershan, E. B. Sirota, and L. B. Sorensen, Phys. Rev. Lett. 52, 356 (1984).

${ }^{3}$ E. B. Sirota, P. S. Pershan, L. B. Sorensen, and J. Collett, Phys. Rev. Lett. 55, 2039 (1985).

${ }^{4}$ E. B. Sirota, P. S. Pershan, S. Amador, and L. B. Sorensen, Phys. Rev. A 35, 2283 (1987).

${ }^{5}$ G. W. Smith, Z. G. Gardlund, and R. J. Curtis, Mol. Cryst. Liq. Cryst. 19, 327 (1973).

${ }^{6}$ G. W. Smith and Z. G. Gardlund, J. Chem. Phys. 59, 3214 (1973).

${ }^{7}$ D. E. Moncton and R. Pindak, Phys. Rev. Lett. 43, 701 (1979).

${ }^{8}$ A. J. Leadbetter, J. C. Frost, and M. A. Mazid, J. Phys. (Paris) Lett. 40, 325 (1979).

${ }^{9}$ A. J. Leadbetter, M. A. Mazid, B. A. Kelly, J. W. Goodby, and G. W. Gray, Phys. Rev. Lett. 43, 630 (1979).

${ }^{10}$ A. J. Leadbetter, M. A. Mazid, and R. M. Richardson, in Proceedings of the Bangalore Conference on Liquid Crystals, 1979, edited by S. Chandreasekhar (Hayden and Sons, London, 1980).

${ }^{11}$ P. A. C. Gane, A. J. Leadbetter, and P. G. Wrighton, Mol. Cryst. Liq. Cryst. 66, 245 (1981).

12J. Doucet and A. M. Levelut, J. Phys. (Paris) 38, 1163 (1977).

${ }^{13}$ A. M. Levelut, J. Doucet, and M. Lambert, J. Phys. (Paris)
35, 773 (1974).

14J. Collett, L. B. Sorensen, P. S. Pershan, R. J. Birgeneau, J. D. Litster, and J. Als-Nielsen, Phys. Rev. Lett. 49, 553 (1982).

${ }^{15}$ J. Collett, L. B. Sorensen, P. S. Pershan, and J. Als-Nielsen, Phys. Rev. A 32, 1036 (1985).

${ }^{16}$ E. B. Sirota, P. S. Pershan, and M. Deutsch, following paper Phys. Rev. A 36, 2902 (1987).

${ }^{17}$ K. Chan, Ph.D. thesis, Harvard University, 1984.

${ }^{18}$ C. Y. Young, R. Pindak, N. A. Clark, and R. B. Meyer, Phys. Rev. Lett. 40, 773 (1978).

${ }^{19}$ M. Born and E. Wolf, Principles of Optics (Pergamon, Oxford, 1975).

${ }^{20}$ J. A. Collett, Ph.D. thesis, Harvard University, 1983.

${ }^{21}$ Committee on Colorimetry, Optical Society of America, in The Science of Color (Crowell, New York, 1953).

${ }^{22}$ A. S. Farber, Ph.D. thesis, Brandeis University, 1985.

${ }^{23}$ D. E. Moncton and G. S. Brown, Nucl. Instrum. Methods 208, 576 (1983).

${ }^{24}$ CPAC Organix, Inc. and Frinton Laboratories, Inc.

${ }^{25}$ P. S. Pershan, G. Aeppli, J. D. Litster, and R. J. Birgeneau, Mol. Cryst. Liq. Cryst. 67, 205 (1981).

${ }^{26} \mathrm{D}$. E. Moncton and R. Pindak, in Proceedings of the Conference on Ordering in Two Dimensions, Lake Geneva, Wisconsin, 1980, edited by S. K. Sinha (North-Holland, Amsterdam, 1980), pp. 83-90.

${ }^{27}$ A. J. Leadbetter, J. P. Gaughan, B. Kelly, G. W. Gray, and J. Goodby, J. Phys. (Paris) Colloq. 40, C3-178 (1979).

${ }^{28}$ J. J. Benattar, J. Doucet, M. Lambert, and A. M. Levelut, Phys. Rev. A 20, 2505 (1979).

${ }^{29}$ P. A. C. Gane, A. J. Leadbetter, J. J. Benattar, F. Moussa, 
and M. Lambert, Phys. Rev. A 24, 2694 (1981).

${ }^{30}$ J. J. Benattar, F. Moussa, M. Lambert, and C. Germain, J. Phys. (Paris) Lett. 42, 67 (1981).

${ }^{31}$ F. Moussa, J. J. Benattar, and C. Williams, Mol. Cryst. Liq. Cryst. 99, 145 (1983).

${ }^{32}$ D. Guillon, A. Skoulios, and J. J. Benattar, J. Phys. (Paris) 47, 133 (1986).

${ }^{33}$ D. R. Nelson and B. I. Halperin, Phys. Rev. B 21, 5312 (1980).

${ }^{34}$ J. J. Benattar, F. Moussa, and M. Lambert, J. Chim. Phys. 80, 99 (1983).

${ }^{35}$ J. Budai, R. Pindak, S. C. Davey, and J. W. Goodby, J. Phys. (Paris) Lett. 45, 1053 (1984).

${ }^{36}$ J. Doucet, P. Keller, A. M. Levelut, and P. Porquet, J. Phys. (Paris) 39, 548 (1978).

${ }^{37}$ E. B. Sirota, J. Collett, P. S. Pershan, and L. B. Sorensen (unpublished).

${ }^{38}$ A. Farber (private communication).

${ }^{39}$ S. C. Davey, J. Budai, J. W. Goodby, R. Pindak, and D. E. Moncton, Phys. Rev. Lett. 53, 212 (1984).

${ }^{40}$ B. M. Ocko, A. R. Kortan, R. J. Birgeneau, and J. W. Goodby, J. Phys. (Paris) 45, 113 (1984)

${ }^{41}$ E. B. Sirota and P. S. Pershan (unpublished). This distortion has also been measured in the $\mathrm{Cr} G$ phases of $90.4,50.6$, and 4-(2'-methylbutyl)phenyl $\quad 4$ '- $n$-octylbiphenyl-4-carboxylate
(8SI) and values of $0.72,0.81$, and $0.38 \%$, respectively, have been obtained for the relative contraction in the direction of tilt. The distortion in the $\mathrm{Cr} J$ phase (crystal analogue of $\mathrm{Sm} I)$ of $p$-hexyloxybenzylidene- $p^{\prime}$-amino-2-chloro- $\alpha$-propylecinnamate, (HOBACPC), 8SI, and 4-(2'-methylbutyl)phenyl $4^{\prime}$-n-octylbiphenyl-4-carboxylate, (80SI) were found to be $0.30,0.30$, and $0.32 \%$, respectively, with the lattice being contracted perpendicular to the direction of tilt.

${ }^{42}$ J. Doucet, A. M. Levelut, and M. Lambert, Phys. Rev. Lett. 32, 301 (1974).

${ }^{43}$ S. Heinekamp, R. A. Pelcovits, E. Fontes, E. Yi Chen, R. Pindak, and R. B. Meyer, Phys. Rev. Lett. 52, 1017 (1984).

${ }^{44}$ D. E. Moncton, R. Pindak, S. C. Davey, and G. S. Brown, Phys. Rev. Lett. 49, 1865 (1982).

${ }^{45}$ D. J. Bishop, W. O. Sprenger, R. Pindak, and M. E. Neubert, Phys. Rev. Lett. 49, 1861 (1982).

${ }^{46}$ P. S. Pershan and J. Als-Nielsen, Phys. Rev. Lett. 52, 759 (1984).

${ }^{47}$ B. M. Ocko, A. Braslau, P. S. Pershan, J. Als-Nielsen, and M. Deutsch, Phys. Rev. Lett. 57, 94 (1986).

${ }^{48}$ B. I. Halperin and D. R. Nelson, Phys. Rev. Lett. 41, 121 (1978).

${ }^{49}$ D. R. Nelson and B. I. Halperin, Phys. Rev. B 19, 2456 (1979). 